\title{
Dislodging anchored diagnoses: an unusual presentation of acute
}

appendicitis

Talal Asif, $\mathrm{MD}^{1}$, Ravali Gummi, MSV${ }^{1}$, Zara Wadood, $\mathrm{MD}^{1}$, Kristy E. Steigerwalt, PharmD ${ }^{1}$, Rebecca R. Pauly, $\mathrm{MD}^{1}$

\section{Author Affiliations:}

1. University of Missouri-Kansas City, Kansas City, Missouri

The authors have no conflicts of interest to disclose.

\section{Corresponding Author:}

Talal Asif, MD

University of Missouri-Kansas City

Kansas City, Missouri

Email: asift@umkc.edu 


\section{Abstract}

Cognitive errors are the most common cause of diagnostic errors in the process of clinical decision making. Recently, there has been an immense focus on the new science of error prevention in health care. Anchored thinking or heuristics is a form of cognitive error that portrays shortcuts or abbreviated thinking in the light of diagnostic uncertainty. The risk is of comfort in a typical scenario and not being able to adapt to a new situation. Here we present an intriguing case of acute appendicitis in a patient who was initially admitted and treated for hypothermia and alcohol withdrawal. As the patient recovered from his withdrawal and neared the end of his hospital stay, he developed persistent leukocytosis, mild metabolic acidosis and vague abdominal discomfort, leading us to pursue further work up resulting in a new diagnosis. If unrecognized, delayed diagnosis and significant harm with potential for rupture of the appendix, peritonitis, significant morbidity and even mortality could have occurred. This case serves as an example of how to avoid errors resulting from heuristic thinking for the reason that incongruity of data was specifically looked for and consequently recognized.

\section{Keywords}

Anchored thinking

\section{Introduction}

Anchored thinking is a concept that describes a cognitive bias whereby physicians link new symptoms and signs in a patient to an existing working diagnosis. It portrays linear thinking that begins with initial management based on the most probable diagnosis followed by strict adherence to that diagnosis. ${ }^{1}$ It is a widely practiced philosophy. Effects of anchoring appear to occur on a subconscious level. ${ }^{2}$ As it is difficult to predict a patient's clinical course, it requires flexibility on the part of the physician especially as patient circumstances change. An astute physician needs to adapt to changing patient situations and respond appropriately which may require a shift from the original diagnosis.

Physicians often have little insight and understanding of the considerable number of biases that influence their thinking. ${ }^{3}$ Given the possibility of missed diagnoses and potential harm to the patient, being mindful of anchoring is an objective approach to improve both quality and safety. With the ability to adjust based on new findings not only will patient care be enhanced but also the cost accrued from missed diagnoses and errors will be lessened. ${ }^{4}$ We present here an awareness of anchored thinking to help avoid cognitive errors and remind us to redirect when the clinical situation changes.

\section{Case Report}

A 32 year old male, with a history of alcohol abuse, was admitted to the ICU with acute ethanol intoxication and hypothermia. He was found unconscious during one of the winter months outside the hospital with emptied alcohol bottles. At presentation, his blood pressure was $144 / 116 \mathrm{mmHg}$, heart rate was $104 / \mathrm{min}$, rectal temperature was $90.3^{\circ} \mathrm{F}$ and respiratory rate was 10/min. He was drowsy but arousable, had cold clammy skin, was not shivering and had an 
abrasion on his right forehead. On physical exam, there were no focal neurological deficits. His cardiovascular, respiratory, abdominal and musculoskeletal exams were unremarkable.

His initial labs revealed a serum alcohol level of $488 \mathrm{mg} / \mathrm{dl}$. CT scan of brain did not reveal an acute intra-cranial process. EKG showed sinus tachycardia and no changes consistent with hypothermia such as prolongation of PR, QRS, QT intervals or Osborn waves. His white cell count was $13300 / \mathrm{cmm}$ with $74.5 \%$ granulocytes. Serum bicarbonate level was mildly decreased to $20 \mathrm{mmol} / \mathrm{l}$ with an anion gap of 19 . His serum electrolytes, CK level and liver enzymes were within normal limits. Urinalysis and urinary drug screen were negative. Patient was placed under a Bair Hugger for rewarming. He was initiated on CIWA protocol and administered intravenous diazepam, thiamine, multivitamins and normal saline. ${ }^{5}$

With treatment, his GCS improved to 15/15 the next day. He began oral intake with regular diet and his CIWA scores decreased from a range of 18-21 to 9-15. His core temperature improved to $98.4^{\circ} \mathrm{F}$ and white cell count improved to $12600 / \mathrm{cmm}$. He was moved to the floor. His CIWA scores declined to zero by the end of day 2 .

Morning labs on day 4 of admission revealed neutrophilic leukocytosis with a rising trend to $24300 / \mathrm{cmm}$ with $86 \%$ granulocytes. Serum bicarbonate level was $19 \mathrm{mmol} / \mathrm{l}$. He also had a slowly rising heart rate over the preceding 24 hours. Figures 1 and $\mathbf{2}$ provide graphs of the trends of vital signs and white cell count during his hospitalization.

Figure 1. This figure illustrates the acute increase in white cell count on days 3 and 4 of admission. Patient underwent urgent appendectomy on Day 4 of hospitalization. After the surgery, his white cell count can be seen returning toward normal.

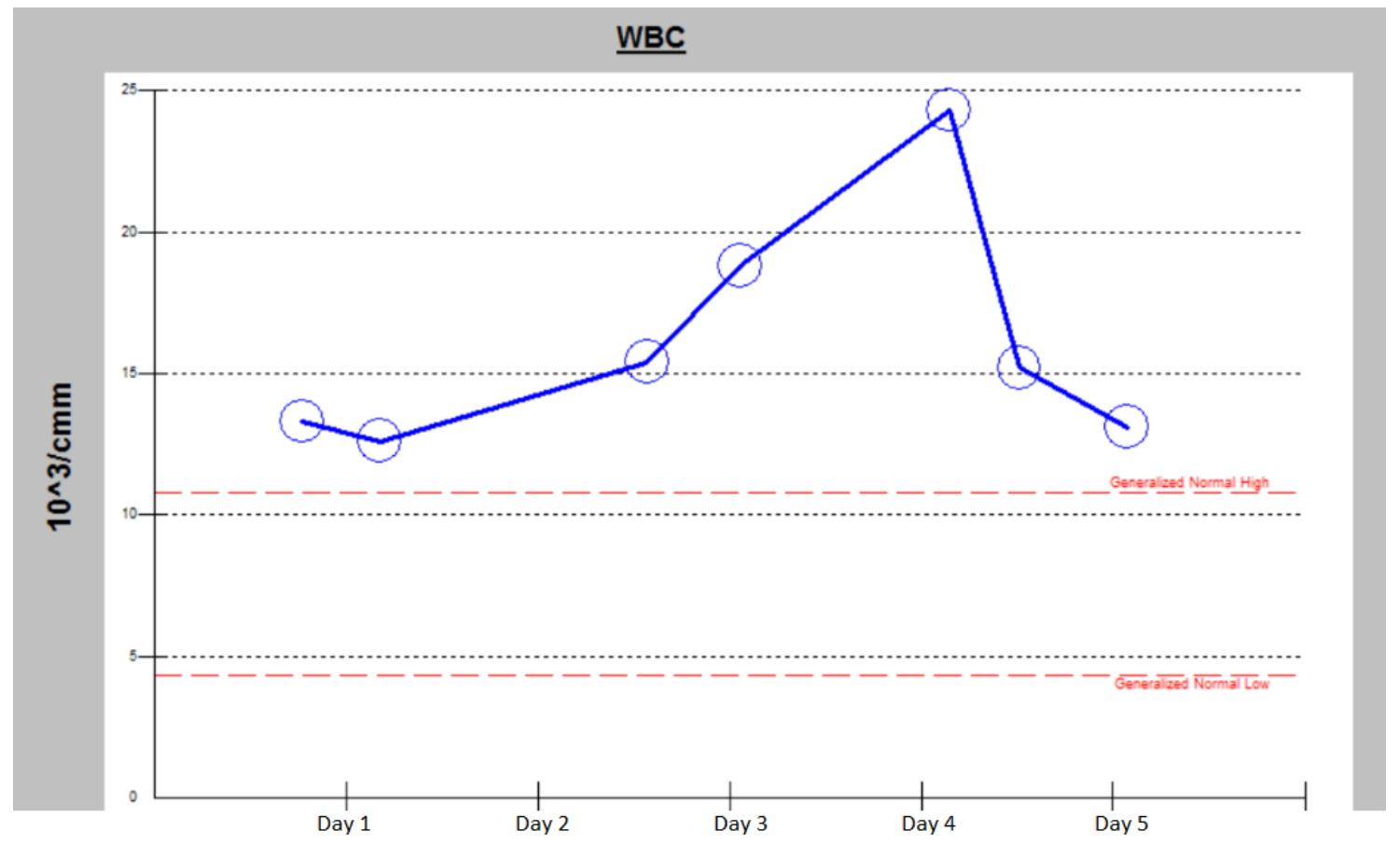


Figure 2: Patient was afebrile after initial hypothermic presentation. Initial tachycardia improved once his alcohol withdrawal symptoms subsided. Heart rate is noted to increase on days 2 and 3 and again post operatively on day 4 of hospitalization.

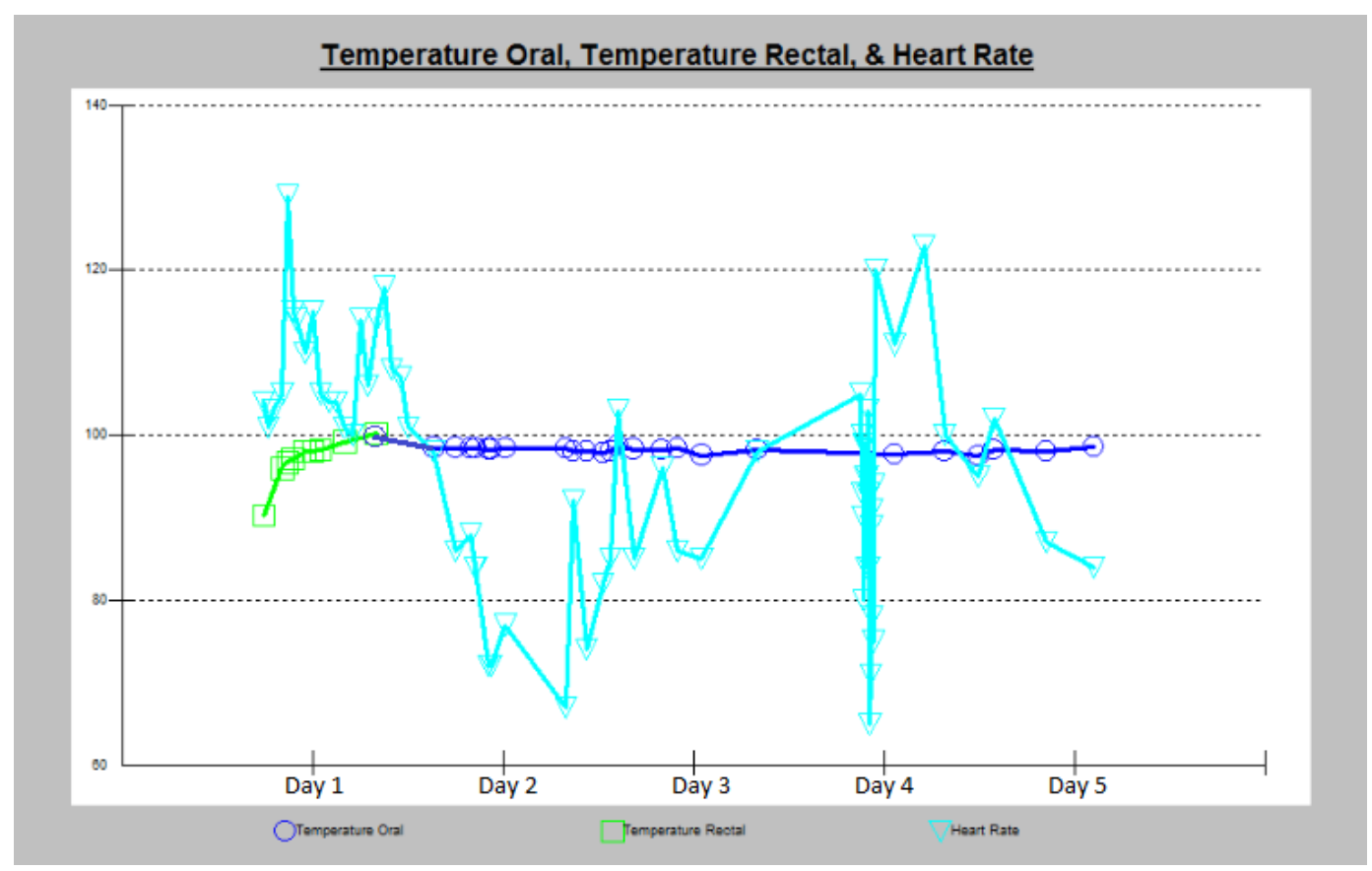

At this juncture, we turned to the fundamentals of clinical evaluation. We performed a detailed history which revealed a symptom of right lower quadrant "uneasiness". Patient denied nausea, vomiting or change in stools. His appetite was good. He did not relate any prior abdominal surgery. His temperature was $97^{\circ} \mathrm{F}$, blood pressure of $119 / 62 \mathrm{mmHg}$, heart rate $78 / \mathrm{min}$ and respiratory rate $14 / \mathrm{min}$. Abdominal examination revealed no distension, bowel sounds positive and mild tenderness to deep palpation in the right lower quadrant. There was no rebound tenderness or guarding. Serum lactate was sent which showed an elevation at $2.8 \mathrm{mmol} / \mathrm{l}$. After an in depth discussion, noting that his current lab abnormalities could not be attributed to his initial diagnosis of withdrawal, we chose to proceed with an ultrasound of the RLQ on lines of a provisional diagnosis of acute appendicitis. ${ }^{6}$ The ultrasound demonstrated a dilated appendix suspicious for acute appendicitis (Figure 3). Patient underwent urgent laparoscopic appendectomy on day 4 of in-patient stay. His serum lactate levels normalized the next day and white cell count started to decline. Surgical pathology proved that he had acute appendicitis without perforation, infarction or necrosis (Figure 4).

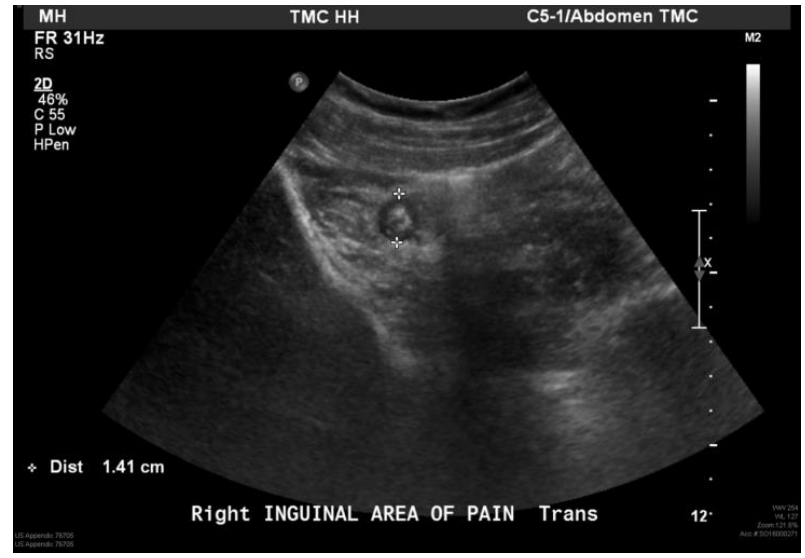

Figure 3. Ultrasound image demonstrates acute appendicitis. The appendix is noncompressible and inflamed. It has fecalith in its lumen. The diameter is $1.41 \mathrm{~cm}$ (up to $7 \mathrm{~mm}$ is regarded as normal). 


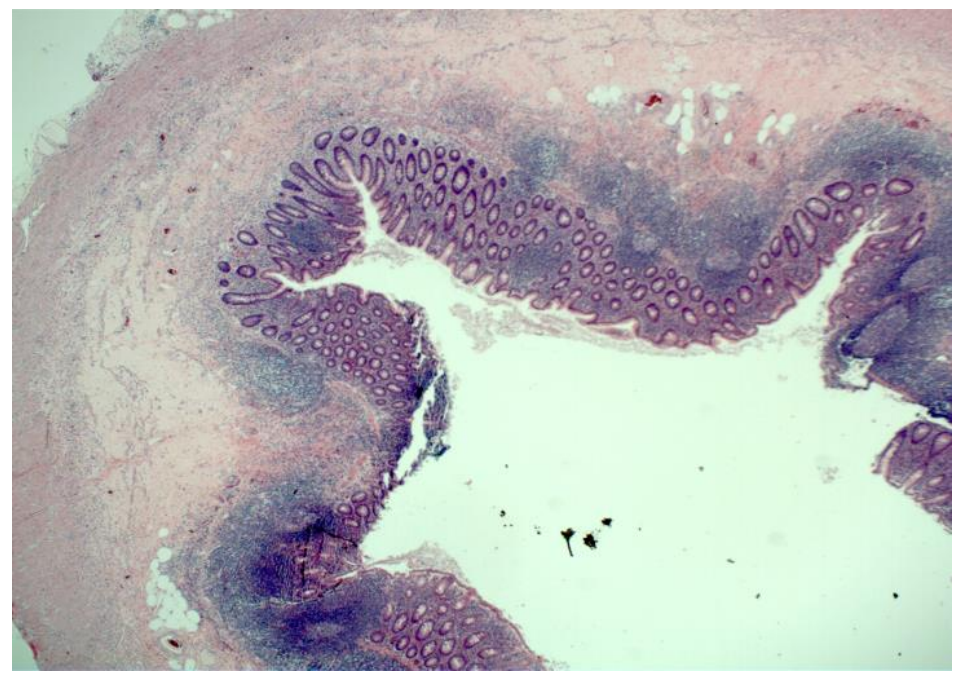

Figure 4. This low powered hematoxylin and eosin (H\&E) stained slide of the appendix shows marked mucosal inflammation and necrosis. It also shows extensive mucosal leukocytic infiltration.

\section{Discussion}

Physicians frequently endorse anchored thinking because it is easier to stay within our comfort zone. $^{7}$ In order to be composed in the face of uncertainty, physicians need to accept that we may need to think beyond what is obvious. When encountered with non-specific symptoms and signs which demonstrate a change regardless of how subtle, we need to be creative and open. If a patient is not responding to treatment, it is imperative to reassess and return to fundamentals of clinical medicine: history and physical exam. We must be ready to admit that the initial diagnosis may no longer be correct. Being stiff may lead to adverse patient outcomes. ${ }^{8} \mathrm{We}$ hypothesize that simply being cognizant of the possibility of anchored thinking will help decrease its rate and improve clinical outcomes.

Important diagnoses may go un-noticed with the tendency to anchor new and unexpected symptoms and signs to the established working diagnosis. This patient had mild clinical features of acute appendicitis which could have been attributed to his original diagnosis. However, the unforeseen seemingly small change in clinical course was discussed on morning rounds with input from several members of the interdisciplinary team. Instead of attributing the new event in our patient to stress from alcohol withdrawal which was our working diagnosis, we avoided anchored thinking and kept our minds open for a different diagnosis. Collaborative effort helped us in escaping from anchored thinking. Delayed or missed diagnosis could have led to significant patient morbidity such as rupture of the appendix.

Familiarity with disease demographics can also be valuable. Since acute appendicitis is more common in young male patients, our index of suspicion was particularly high. The knowledge of disease epidemiology remains an important tool in clinical decision making and formulating a differential diagnosis enabling a more integrative approach. Knowing the pretest probability based on these demographics also helps in appropriate utilization of diagnostic tests and resources. 
This case further demonstrates that it is crucial to be observant of trends in clinical and biochemical parameters during a patient's hospitalization. Even apparently small changes may reflect important underlying pathology. For instance, in our patient, leukocytosis, mild acidosis, and a slowly rising heart rate with the vaguest new symptom of abdominal uneasiness led to the final diagnosis. Our patient did not have the typical features of severe abdominal pain, nausea, vomiting, fever, chills or change in bowel pattern most frequently associated with acute appendicitis. ${ }^{9}$ By dislodging anchored thinking, we were able to contribute to the betterment of our patient, a goal which will remain the center of our practice. 


\section{References}

1. Chapman GB, Johnson EJ. Incorporating the irrelevant: anchors in judgments of belief and value. In: Gilovich T, Griffin DW, Kahneman D, editors. Heuristics and biases: the psychology of intuitive judgment. Cambridge (UK): Cambridge University Press; 2002. p. 120-38.

2. Wilson TD, Houston CE, Etling KM, Brekke N. A new look at anchoring effects: basic anchoring and its antecedents. J Exp Psychol Gen. 1996;125(4):387-402.

3. $\quad$ Epstein RM. Mindful practice. JAMA. 1999;282(9):833-9.

4. Milkman KL, Chugh D, Bazerman MH. How can decision making be improved? Perspect Psychol Sci. 2009;4(4):379-83.

5. Sullivan JT, Sykora K, Schneiderman J, Naranjo CA, Sellers EM. Assessment of alcohol withdrawal: the revised clinical institute withdrawal assessment for alcohol scale (CIWA-Ar). Br J Addict. 1989;84(11):1353-7.

6. Mazzei MA, Guerrini S, Cioffi Squitieri N, Cagini L, Macarini L, Coppolino F, et al. The role of US examination in the management of acute abdomen. Crit Ultrasound J. 2013;5 Suppl 1:S6.

7. Sinclair D, Croskerry P. Patient safety and diagnostic error: tips for your next shift. Can Fam Physician. 2010;56(1):28-30.

8. Misch, DA. A piece of my mind. I feel witty, oh so witty. JAMA. 2016;315(4):345-6.

9. Wray CJ, Kao LS, Millas SG, Tsao K, Ko TC. Acute appendicitis: controversies in diagnosis and management. Curr Probl Surg. 2013;50(2):54-86. 